\title{
Coronary Embolization Caused by Pleomorphic Lung Carcinoma
}

\author{
Tadashi Murai ${ }^{1}$, Taishi Yonetsu ${ }^{1}$, Mitsuaki Isobe ${ }^{2}$ and Tsunekazu Kakuta ${ }^{1}$
}

\begin{abstract}
A 73-year-old man who had been transferred to our emergency room due to sudden chest pain was diagnosed with ST-segment elevation myocardial infarction (STEMI). Primary percutaneous coronary intervention was performed. A long, white object which looked like a parasitic worm was retrieved via intracoronary aspiration and revascularization was successfully completed. Contrast computed tomography revealed a huge $7 \times 6$ $\mathrm{cm}$ mass in the right upper pulmonary lobe with direct pulmonary vein invasion. Histopathologic examination of the aspirated coronary object revealed pleomorphic lung carcinoma. This is an unusual case of STEMI caused by lung tumor embolization via direct pulmonary vein invasion to the left side of the heart.
\end{abstract}

Key words: acute myocardial infarction, tumor embolization, lung cancer, intracoronary aspiration, optical coherence tomography

(Intern Med 55: 3607-3609, 2016)

(DOI: 10.2169/internalmedicine.55.7571)

A 73-year-old man who had been transferred to our emergency room due to sudden chest pain was diagnosed with ST-segment elevation myocardial infarction (STEMI). He had no coronary risk factors except for a history of smoking for 50 years. Primary percutaneous coronary intervention was performed, and a long, string-shaped contrast defect was observed in the left anterior descending artery (Fig. 1A). Optical coherence tomography (OCT) imaging revealed an odd, smooth-surfaced 50-mm long object (Fig. 1B). A long, white object which looked like a parasitic worm was retrieved via intracoronary aspiration and revascularization was successfully completed since neither plaque rupture or the presence of thrombus was detected by OCT (Fig. 1C).

On the eleventh hospital day, transthoracic echocardiography revealed a new floating object in the left atrium. Contrast computed tomography revealed a huge $7 \times 6 \mathrm{~cm}$ mass that was recognized at admission in the right upper pulmonary lobe with direct pulmonary vein invasion (Fig. 1D). Although the bronchoscopic findings were negative for a lung tumor, a histopathologic examination of the aspirated coronary object revealed pleomorphic lung carcinoma (Fig. 2). The patient was diagnosed with STEMI due to coronary em- bolization associated with lung carcinoma.

Brain MRI revealed that the patient had an asymptomatic cerebral infarction in the right frontal lobe and a small parietal lobe lesion was diagnosed to be metastasis. Given all these observations, he was diagnosed with right lung carcinoma, stage IV (c-T2bN2M1b). He was treated according to the chemotherapy regimens (carboplatin \& paclitaxel) known to be effective for the treatment of advanced nonsmall cell lung cancer. He responded to the treatment and showed a good clinical course afterwards (Fig. 3). Chemotherapy with a total of 9 cycles of the same regimen was used and the carcinoma progression was suppressed. He has been doing well for more than two years of PCI and has been followed-up regularly.

This is an unusual case of STEMI caused by lung tumor embolization via direct pulmonary vein invasion to the left side of the heart. To the best of our knowledge, this is the first case report of coronary embolization caused by pleomorphic lung carcinoma.

The authors state that they have no Conflict of Interest (COI).

The Internal Medicine is an Open Access article distributed under the Creative

${ }^{1}$ Department of Cardiology, Tsuchiura Kyodo General Hospital, Japan and ${ }^{2}$ Department of Cardiovascular Medicine, Tokyo Medical and Dental University, Japan

Received for publication April 12, 2016; Accepted for publication May 5, 2016

Correspondence to Dr. Tsunekazu Kakuta, kaz@joy.email.ne.jp 

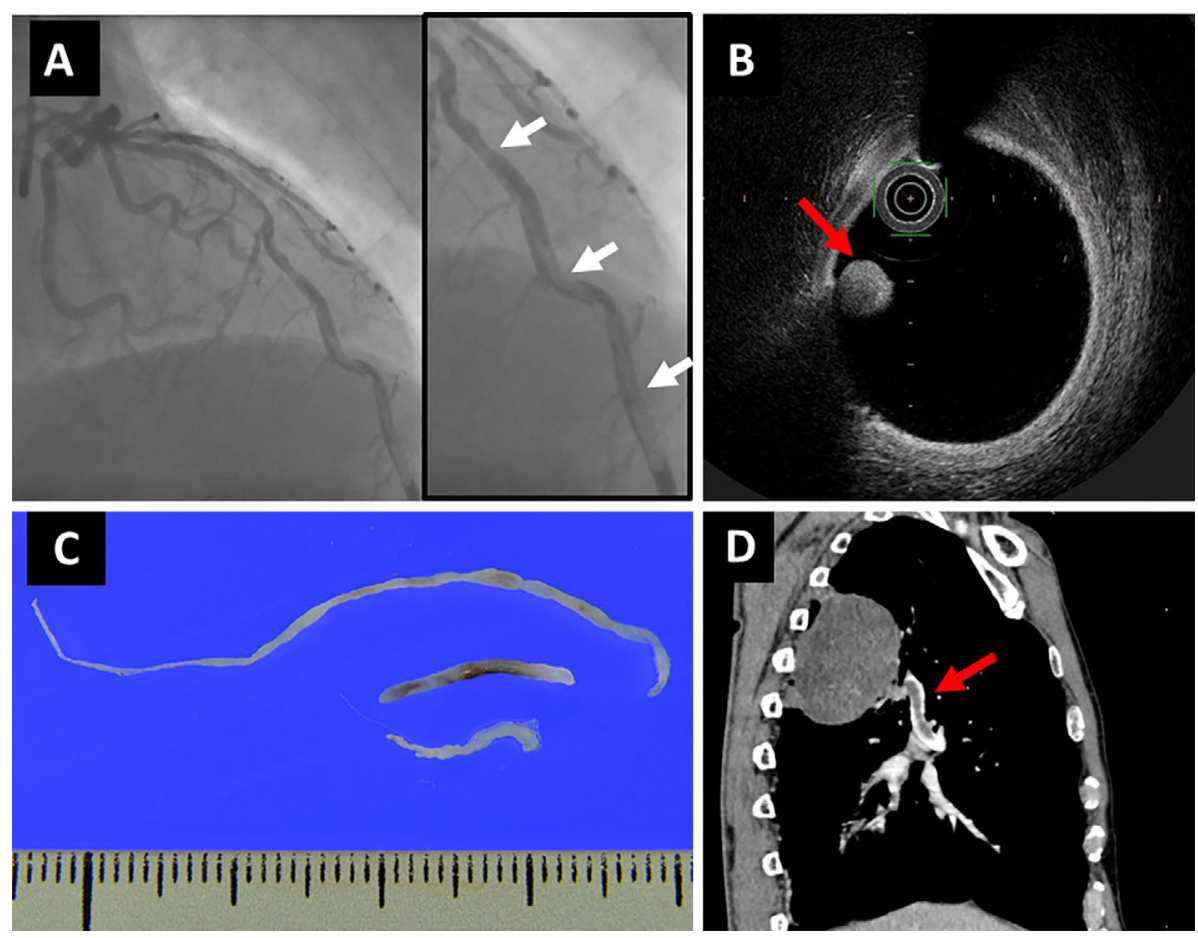

Figure 1. A: Emergency coronary angiography demonstrating a long, string-shaped defect in the left anterior descending artery (white arrow). B: Optical coherence tomography image showing an odd, smooth-surfaced and long object (red arrow). C: The object retrieved via intracoronary aspiration. D: Contrast computed tomography revealed a huge $7 \times 6 \mathrm{~cm}$ mass in the right upper pulmonary lobe with direct pulmonary vein invasion (red arrow).

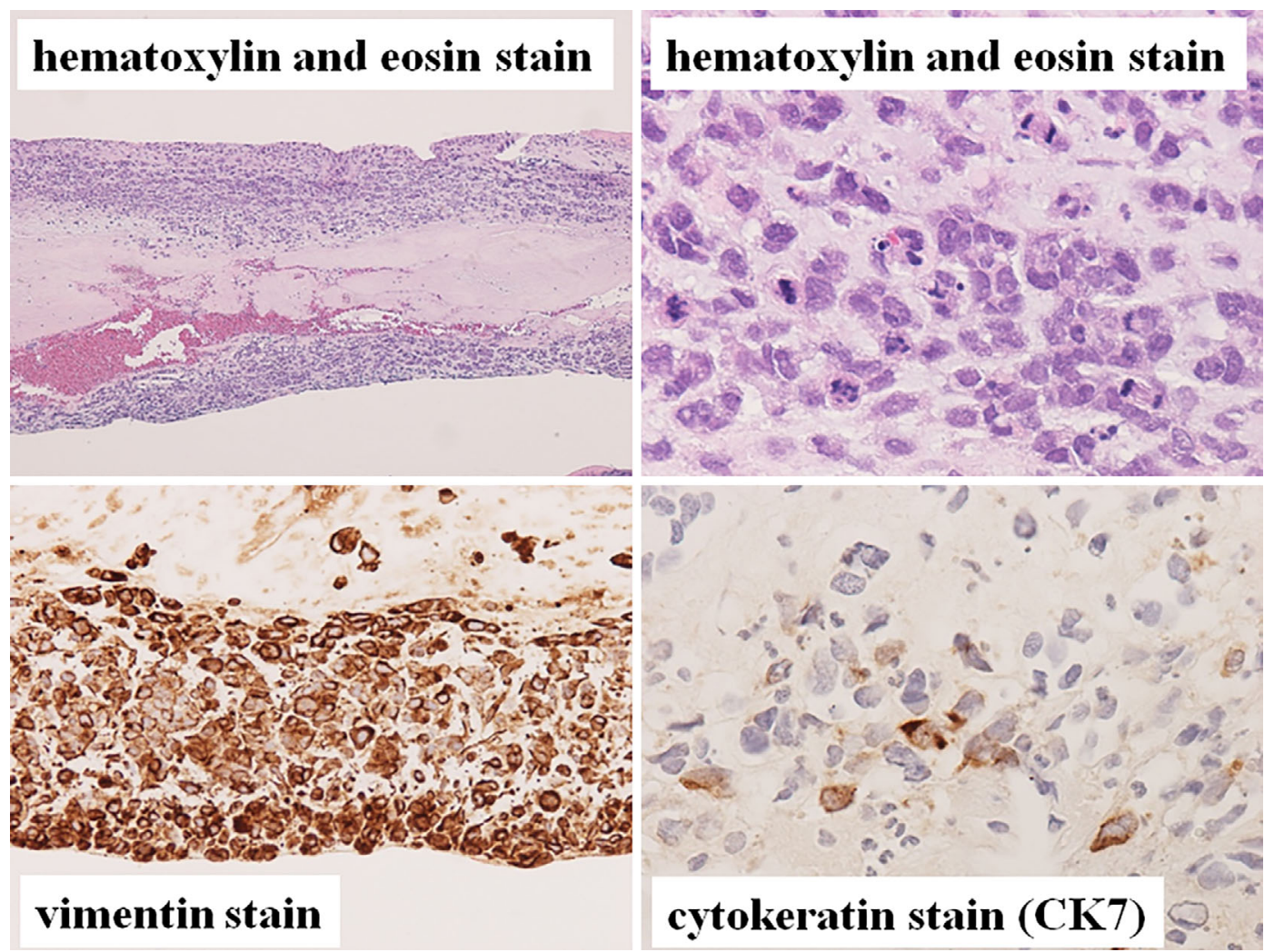

Figure 2. Histopathologic examination of the aspirated coronary object revealed pleomorphic lung carcinoma. Immunohistochemically, tumor cells were positive for both cytokeratin and vimentin. 

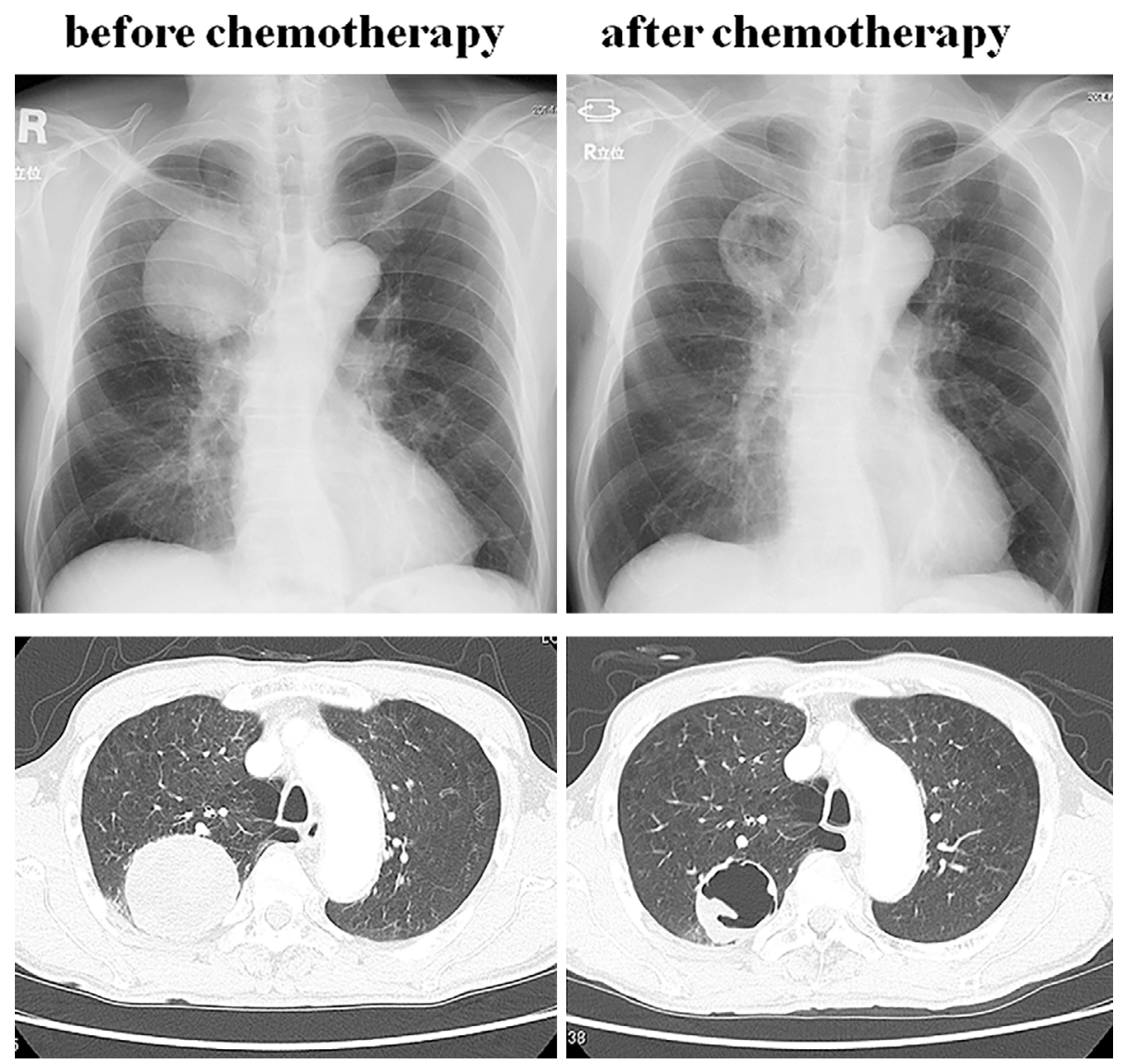

Figure 3. Chest X-ray and computed tomography before and after chemotherapy.

Commons Attribution-NonCommercial-NoDerivatives 4.0 International License. To view the details of this license, please visit (https://creativecommons.org/licenses/

(C) 2016 The Japanese Society of Internal Medicine http://www.naika.or.jp/imonline/index.html 